\title{
Biomechanical Analysis of Gait Adaptability in Parkinson's Disease
}

\author{
Mareike A. Schwed ${ }^{*}, 1$, Tobias Getrost ${ }^{1}$, Dietmar Schmidtbleicher ${ }^{1}$ and Christian T. Haas ${ }^{2}$ \\ ${ }^{1}$ Institute of Sports Sciences J.W. Goethe-University of Frankfurt/Main Ginnheimer Landstr., 3960487 Frankfurt am \\ Main, Germany \\ ${ }^{2}$ Hochschule Fresenius, University of Applied Sciences, Faculty of Health Limburger Straße 265510 Idstein, Germany
}

\begin{abstract}
Parkinson's Disease (PD) subjects are less able to adapt gait to fluctuating motor demands in daily life situations than the healthy elderly, e.g. in crowded places, crossing the road, and starting or stopping at traffic lights. Several studies proved gait abnormalities in PD. However, to our knowledge there is currently no biomechanical test that deals with the ability in gait adaptation. The aim of this study was to develop and evaluate a new biomechanical test which proves the adaptability of gait to fluctuating external conditions.

In order to have a reproducible, quantitative gait-test, a treadmill was used, accessed by a special software. The so called adaptability-of-gait test (AOG) changed the treadmill's velocity ballistically and unpredictably for the test subjects. 49 PD-subjects and 10 age-matched controls (HC) participated in the study. In order to subdivide PD subjects in homogeneous groups, we used a cluster analysis. In a first-step of evaluation we focused on differences between PD with moderate (PDM) and severe disease stages (PDS) and HC and examined correlations according to existing, valid tests, e.g. Unified Parkinson Disease Rating Scale (UPDRS) subscales, clinical and biomechanical gait-assessments.

Results showed significant differences in gait-adaptation between the groups. Severe PD had a worse gait adaptation compared to PD moderate and HC. Correlation analysis of the PD sample showed significance differences between the AOG-test and bradykinesia and facial expression, but no significances differences according to conventional clinical gait assessments, e.g. Webster gait-test. We conclude that the AOG-test has potentials to identify a new gait performance: the adaptation of gait.
\end{abstract}

Keywords: Adaptation of gait, biomechanic test, bradykinesia, CPG,gait-test, Parkinson's disease, treadmill.

\section{INTRODUCTION}

Effective and efficient rehabilitation is based on reliable and valid knowledge of the system parameters (pathology and treatment) that influence motor disturbances. Since gait disturbances in Parkinson's disease (PD) are evident and have a strong impact on quality of life, it is of crucial importance to understand motor control of gait. PD - as one of the most common neurodegenerative diseases [1] - is clinically characterized by the onset of unilateral symptoms with ongoing progression. The cardinal symptoms are resting tremor, muscular rigidity, bradykinesia and postural impairments [2]. Many studies analyzed the gait pattern in PD subjects compared to healthy controls in detail [3]. PD gait is - compared to healthy age-matched subjects characterized as a slowed gait-speed, with shortened, shuffling, wide based steps, a stooped posture, and a reduced arm swing [4, 5]. Spatiotemporal analysis showed a protracted bipedal phase, a higher cadence and exceeded gait variability as additional pathological signs according to the gait-cycle [3, 6-11].

To quantify kinetic and kinematic gait parameters, different biomechanical methods were used, e.g. a thread-

*Address correspondence to this author at the Institute of Sports Sciences J.W. Goethe-University of Frankfurt/Main Ginnheimer Landstr., 3960487 Frankfurt am Main, Germany; Tel: +49 (0) 69 - 798245 23; Fax: +49 69798245 74; E-mail: m.schwed@sport.uni-frankfurt.de pulley system, at which threads are attached by Velcro straps to each foot of a subject $[6,12,13]$; force plates embedded in walkways [14] or in a treadmill [15, 16]; 3-D motion analyzing systems [14]; a clinical stride analyzer [7, 14, 17, 18]; force-sensitive shoe-soles [10, 19]; and pressuresensitive insoles $[9,20,21]$.

These methods are also used to get insights into PD pathology. Since the beginning of the systematic gaitanalyses in PD, it has been well known that most locomotor parameters are related to the Hoehn and Yahr (H\&Y) stages described in the clinical classification $[4,5]$. In the early stages of PD, patients' gait already shows a tendency of a reduced cadence and walking speed, which is associated with the cardinal symptom bradykinesia [3]. Coevally gait impairments occur obviously in transitional conditions, e.g. turning during gait [22]. Winogrodzka et al. showed that rigidity and bradykinesia limited gait-parameters significantly [23]. Several studies reported that PD subjects compensate their impaired stride length with a higher step frequency, a parameter that is not impaired in PD-gait $[6,9$, 17, 24]. All or some of these biomechanical gait parameters may influence the performance in gait-adaptation.

Even if there are several focuses in PD gait studies and the existing methods are proved well, they still cover only a small range of situations and motor demands [3, 14, 25]. In most biomechanical and clinical gait analyses, PD subjects choose their gait parameters themselves (e.g. velocity, step- 
length) or walk with external triggered but constant, chosen velocity. Consequently dynamic requirements, e.g. gait adaptability are low in these test procedures. Various gait studies on the walkway confirmed independently that PDpatients preferred walking speeds between 3.0 and $3.5 \mathrm{~km} / \mathrm{h}$ $[3,26,27]$. However, several gait-demands take place at slower walking speeds. For example, falls often occur at home and are caused by sudden changes in posture, turning movements of the trunk or by dual tasks [28]. These home activities are accomplished at slower walking speeds [29]. Moreover, gait has to be adapted to urban environmental conditions e.g. pedestrian zones, traffic lights etc. and requires different velocity-ranges. These everyday situations have to be adapted according to external conditions and are characterised by gait parameters that are not chosen freely. Thus, the gait parameters like gait velocity, step frequency or stride length are not constant. They underlie a dynamic process with sudden and unpredictable velocity changes like acceleration, deceleration, stopping and initiating gait. Regarding gait analysis, the diversification of existing clinical and biomechanical methods is required [3]. Even if it is well known that PD subjects have serious exertions with gait adaptability, to our knowledge valid tests are missing to date.

Therefore, the aim of this study was to develop and evaluate a method of biomechanical gait analysis, which analyses the adaptability in gait to fluctuating external conditions. Therefore we developed the AOG-test (AOG stands for adaptability of gait).

The test procedure was based on the following preconditions: 1) test-reliability, -objectivity and -accuracy; 2) the test results have to be accessible immediately; 3 ) the testing procedure should ensure a high patient safety; 4) the test procedure should be usable in clinical infrastructures with limited space and human resources. In order to realise the above mentioned, we decided to use a treadmill for the AOG-test, keeping in mind that the validity of the gaitpattern on the treadmill is diversely discussed [30, 31]. Several significant differences are reported between overground- and treadmill walking [30], e.g. EMG profiles [32-34], ground reaction forces [35, 36] or inter limb synchronicity [30]. Carpinella et al. observed that treadmill walking might be regulated through a different 'motor set' [30]. In addition, treadmill walking might require greater attentional demands and a more constant supraspinal control [37]. Other authors argue that the kinematic patterns [32, 34, 38] and gait biomechanics [36] are highly similar between both walking conditions and therefore treadmill walking can be transferred to overground walking.

To evaluate the AOG-test, we focused on gait differences between PD and healthy controls (HC). Additionally, we analyzed connections between the new AOG-parameters with established biomechanical and clinical gait parameters and the cardinal symptoms.

\section{METHODS}

\subsection{Subjects}

We recruited subjects from the Parkinson Clinic Bad Nauheim (Germany) and several PD support groups. The ethic committee of the Justus Liebig University of Giessen
(Germany) granted ethical approval of the study. All included subjects had a diagnosis of idiopathic PD, disease severity of Hoehn and Yahr stage I-III (H\&Y) and were able to walk on a treadmill. We performed all evaluations while the subjects were pharmaceutically "on". Volunteers were screened in a personal interview and a neurological examination. Age-matched control subjects were recruited from the community. PD subjects' marriage partners were also required.

Descriptive information was examined for subjects' characteristics (age and sex) and disease severity. With respect to the heterogeneous structure of $\mathrm{PD}$, we used cluster analysis (see statistical analysis) in order to subdivide groups. We compared the performance in adaptation of gait between these groups, conctretly named PD moderate (PDM) and PD severe (PDS) (Tab. 1). In correlation analysis we focused on the pathological differences of gait in PD, assuming, that $\mathrm{HC}$ results would have ceiling effects in the tests e.g. Webster-test, biomechanical gait analysis.

\subsection{Clinical Assessments}

\subsubsection{UPDRS Scales}

The Unified Parkinson's Disease Rating Scale (UPDRS) is the standard and the most commonly used clinical assessment in PD [39]. The four subscales focus on I mentation, behaviour and mood; II activities of daily life (ADL); III: clinical assessed motor score and IV: H \& Y stadium and assessment of other complications according to advanced disease.

Subscore indices of motor function were determined from the UPDRS motor score (III) [39], to compare standardised clinical assessments with the AOG-test results.

- Subscore cranial includes the items 'speech' and 'facial expression',

- $\quad$ subscore tremor includes the items 'tremor at rest' and 'action or postural tremor of hands',

- $\quad$ subscore rigidity includes the items 'rigidity' and 'finger taps',

- subscore bradykinesia includes the items 'hand movements', 'rapid alternating movements of hands', 'leg agility' and 'body bradykinesia' and 'hypokinesia',

- $\quad$ subscore gait and posture include the items 'arising from chair', 'posture', 'gait' and 'postural stability'.

\subsubsection{Webster Gait-Test}

Patients were asked to walk a distance of $12 \mathrm{~m}$ in their common walking velocity, and a distance of $24 \mathrm{~m}$ with a turn after $12 \mathrm{~m}$. The time was taken for the $12 \mathrm{~m}$ distance and the $24 \mathrm{~m}$ distance [40].

\subsection{Biomechanical Gait Assessment}

\subsubsection{Spatiotemporal Gait Assessment}

To get spatiotemporal gait-parameters in constant gait velocities, a treadmill with two independently functioning force platforms was used. The treadmill was used for velocity-standardization and comparison to the AOG-test ( $v$ $[\mathrm{km} / \mathrm{h}]: 1.5,1.8,2.1,2.4,2.7$ and 3.0). Vertical groundreaction-force (GRF) of left and right foot-contact gave 
Table 1. Subject Characteristics

\begin{tabular}{|c|c|c|c|c|c|c|c|c|c|}
\hline & $\mathbf{n}$ & Age (y) & Sex (f/m) & UPDRS & UPDRS Part III & H \& Y & L-Dopa (Yes/No) & LED (mg/d) & Disease Duration (m) \\
\hline \hline PDM & 16 & $59.06(8.02)$ & $6 / 10$ & $27.66(5.82)$ & $14.41(4.34)$ & $2.06(.61)$ & $10 / 6$ & $251.25(252.21)$ & $56.1(35.7)$ \\
\hline PDS & 20 & $63.1(8.58)$ & $13 / 7$ & $42.48(6.88)$ & $25.78(3.92)$ & $2.43(.37)$ & $12 / 8$ & $201.25(198.1)$ & $62.9(59.1)$ \\
\hline HC & 8 & $63.88(9.0)$ & $4 / 4$ & & & & & & \\
\hline
\end{tabular}

Values are means $( \pm$ SD). UPDRS: Unified Parkinson Disease rating Scale; H \& Y: Hoehn \& Yahr; L-Dopa: Levodopa; LED: Levodopa Dose

information about parameters e.g. stride-length, stride time, double-stance time, stance- and swing time. The variables stride-length and stride time are intercorrelated, because of the constant velocity of the treadmill. Spatiotemporal gaitparameters were quantified using GRF-graphs within 10 strides of 30 seconds. Stride time and stride-length were quantified using spectral analysis (FFT, Hamming-window).

\subsubsection{The AOG-Test}

\subsubsection{AOG-Test Apparatus}

In order to realise the test setting, we used a slat-style treadmill with a ball bearing design (Woodway ${ }^{\circledR}$ ). Compared to a conventional treadmill, this treadmill accelerates immediately and with low friction. The subjects had the task to walk in a constant position that was marked at the treadmill's handrail (Fig. 1a). They were instructed that the treadmills velocity will change unpredictably. The feasible velocities were tested before (see 2.3.1), an incremental encoder with a cable-belt-system was used to measure the subject's position (Fig. 1b, c). We used a standardised test setting with a self-programmed software in order to transmit data bit-serially to the treadmill's serial-interface. The AOGtest had a significantly good test-retest reliability between two independent gait trials in $\mathrm{HC}(\mathrm{r}=0.747$; $\mathrm{p}<0.05)$.
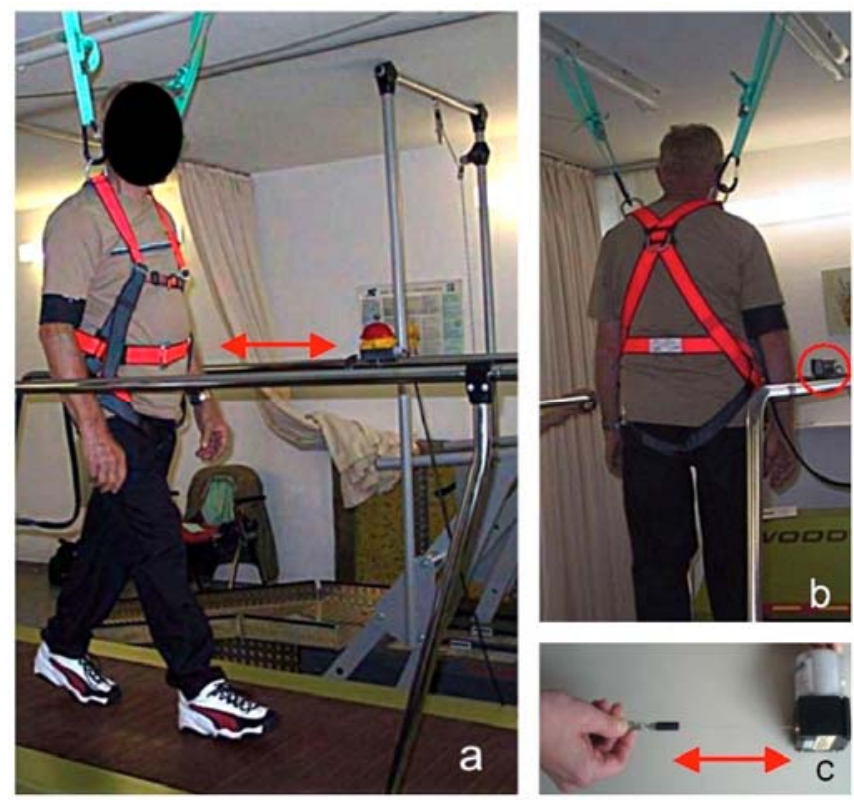

Fig. (1). (a, b, c) PD patient walking on the treadmill with security belt, a. Side view, b. back view, c Incremental encoder.

The test-velocities were set in the range between 1.2 and $3.8 \mathrm{~km} / \mathrm{h}$. We decided to change the velocity every 8 -seconds according to the following assumptions: the PD subjects should be able to react adequately to new gait conditions. If velocity would change more often, the reaction pattern might be biased, since velocity-compensation strategies eg. Bradykinesia or dyskinesia could superpose each other, e.g. studies found hyperstability of walking coordination that leads to difficulties in adapting to a single velocity change during gait on a treadmill [45]. These sequential operations might be overlapping [41]. The total testing-time was set to 5.30 minutes, to avoid physical fatigue. A velocity increment $>8$ Seconds might have started external cueing effects [19] and the total testing time would have risen. The type of modification (acceleration or deceleration) and the different velocity changes were standardised and called AOG-test (Fig. 2a, b, upper trace). These modifications could not be anticipated by the subjects.

\subsubsection{AOG Data Analysis}

To analyze the data of the AOG-test, the following presumptions were taken. In a cybernetic view the overall performance can be regarded as a linear velocitycompensation in terms of

velocity-changing - sensor identification - motor reaction - ready for the next perturbation.

A limitation of this view is that the AOG-performance is neither a single, nor an ad-hoc reaction. In fact, a linear analyzing-strategy would distort the presented phenomenon. Spectral analysis (FFT, Hamming window) was used, to analyze patterns in the subjects-position changes due to the fluctuating velocities. We quantified the spectral-diagram to enable insights in the different operations, e.g. overcompensatory gait conclusions, delayed compensation or long term compensation.

For quantifying the overall results (AOG-all) the whole position-over-time-graph of the subjects was taken (Fig. 2a, b, lower trace) and integrated after FFT. For more detailed information about time-related performance in gait adaptability, we split the FFT-graph into four frequency bands to relate time dependent differences of gait-adaptation (AOG I-IV, Fig. 3A-C). Therefore we divided the upper spectral graph into four sub sequences: AOG I reflects 7-18 velocity changes in the test, AOG II reflects 3-7 changes, AOG III reflects 2-3 changes and AOG IV reflects 1-2 changes. For example, a high value in AOG I represents a more frequent change in position of the subject over the whole test. A higher value in AOG IV represents changes of the subject nearly every 8-seconds. Data acquisition and evaluation, e.g. spectral analysis were conducted with DasyLab $8.0^{\circledR}$. 

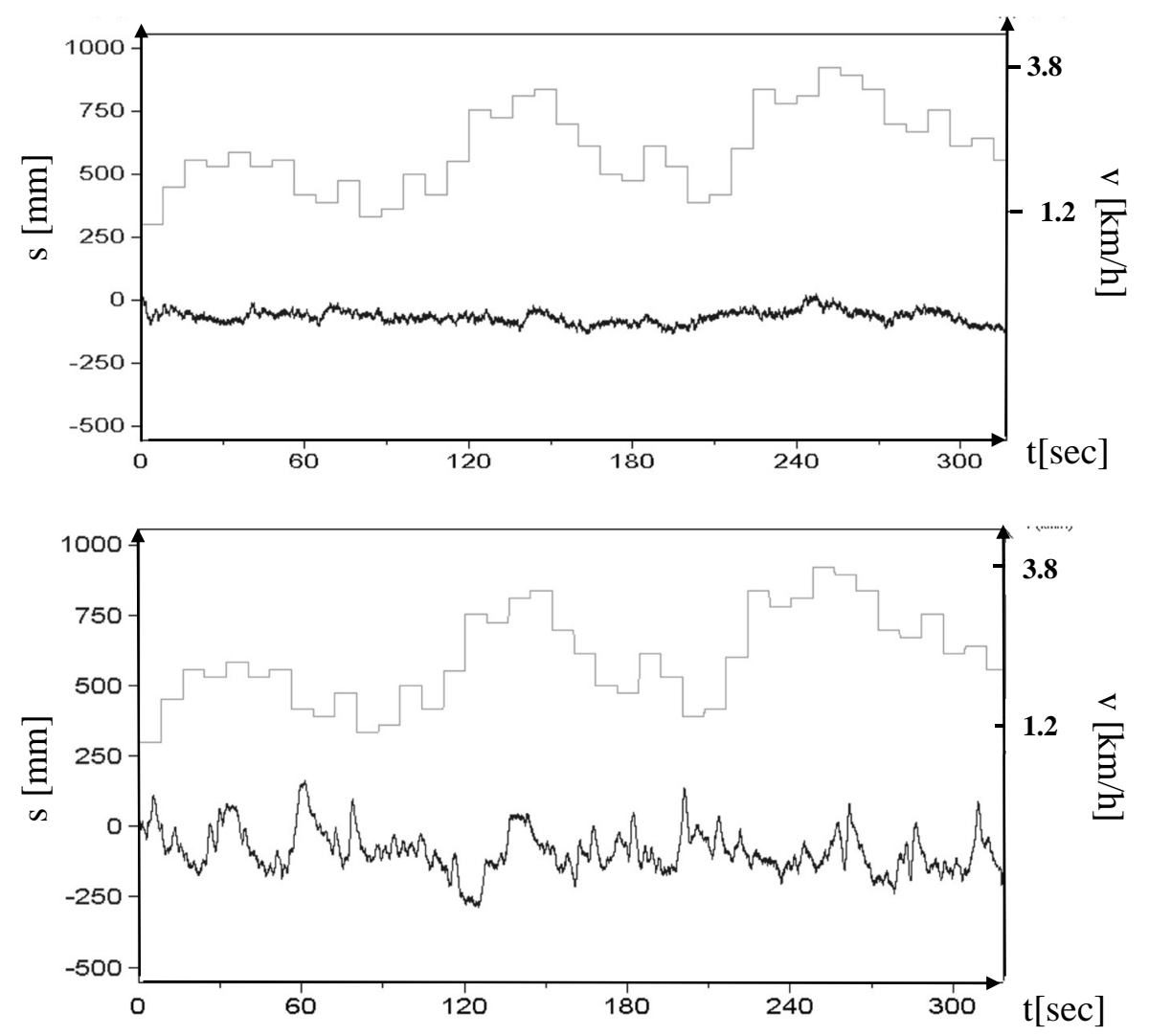

Fig. (2). (a, b) Different examples of gait adaptation at the AOG-test: Fig. (2a, b) show different gait adaptations according to the AOG test. The upper traces show the treadmill's velocity, the lower traces show the subject's position on the treadmill. The subject's position in graph a) represents a good gait-adaptability. The subject in graph b) represents a higher variance in gait-adaptability, thus the subject's position varies between plus/minus $20 \mathrm{~cm}$ according to the subject's starting-position.

\subsection{Statistic Analysis}

\subsubsection{Cluster Analysis}

Calculations of statistical data are based on an initial cluster analysis (squared Euclidian distance, ward method) in order to split patients into homogeneous groups. The factors for division were the 14 single variables of the UPDRS motor examination: speech, facial expression, tremor at rest, action or postural tremor of hands, rigidity, finger taps, hand movements, rapid alternating movements of hands, leg agility, body bradykinesia, hypokinesia, arising from chair, posture, gait and postural stability. The resulting groups were divided in parkinson disease moderate (PDM) and parkinson disease severe (PDS) and compared to healthy controls (HC).

\subsubsection{ANOVA}

Normality of the AOG-data was proven by using the nonparametric Kolmogorov-Smirnov test (K-S test). A one-way analysis of variance (ANOVA) and post-hoc analysis (LSD) were calculated to get information about group differences.

\subsubsection{Correlation Analyses}

A correlation analyses was done only for the PD group data.

Pearson's correlation coefficient was used to evaluate the association between dynamic gait variables and AOG frequency and non frequency-weighted parameters.
Spearman's correlation coefficient was used to evaluate the association between AOG parameters and the clinical features. A $p$-value of less than 0.05 (two-tailed) was considered statistically significant. All statistical analyses were performed using SPSS $19.0^{\circledR}$.

\section{3, RESULTS}

All subjects performed the task without complications. Subjects were excluded from data analysis, when touching the handrail for safety reasons (4 PD), in sudden Off-state (1 PD); and because of several reasons (6 PD; $2 \mathrm{HC}$ ). The data of thirty-eight PD- and eight HC subjects were analyzed.

\subsection{ANOVA Results}

\subsubsection{AOG-Overall}

ANOVA detected significant group differences for AOGall results. Post-hoc analysis showed that PDS performed significantly worse compared to PDM and HC. Between PDM and HC no statistical differences became evident (Fig. 4).

\subsubsection{AOG-Overall}

Table 2 summarizes the data of the frequency-weighted performance in AOG I-IV. ANOVA showed significant differences in AOG II and a statistical trend in AOG III between the groups. Post-hoc analysis showed that PDS performed significantly worse compared to PDM in AOG II and in AOG III. PDS performed significantly worse than HC 


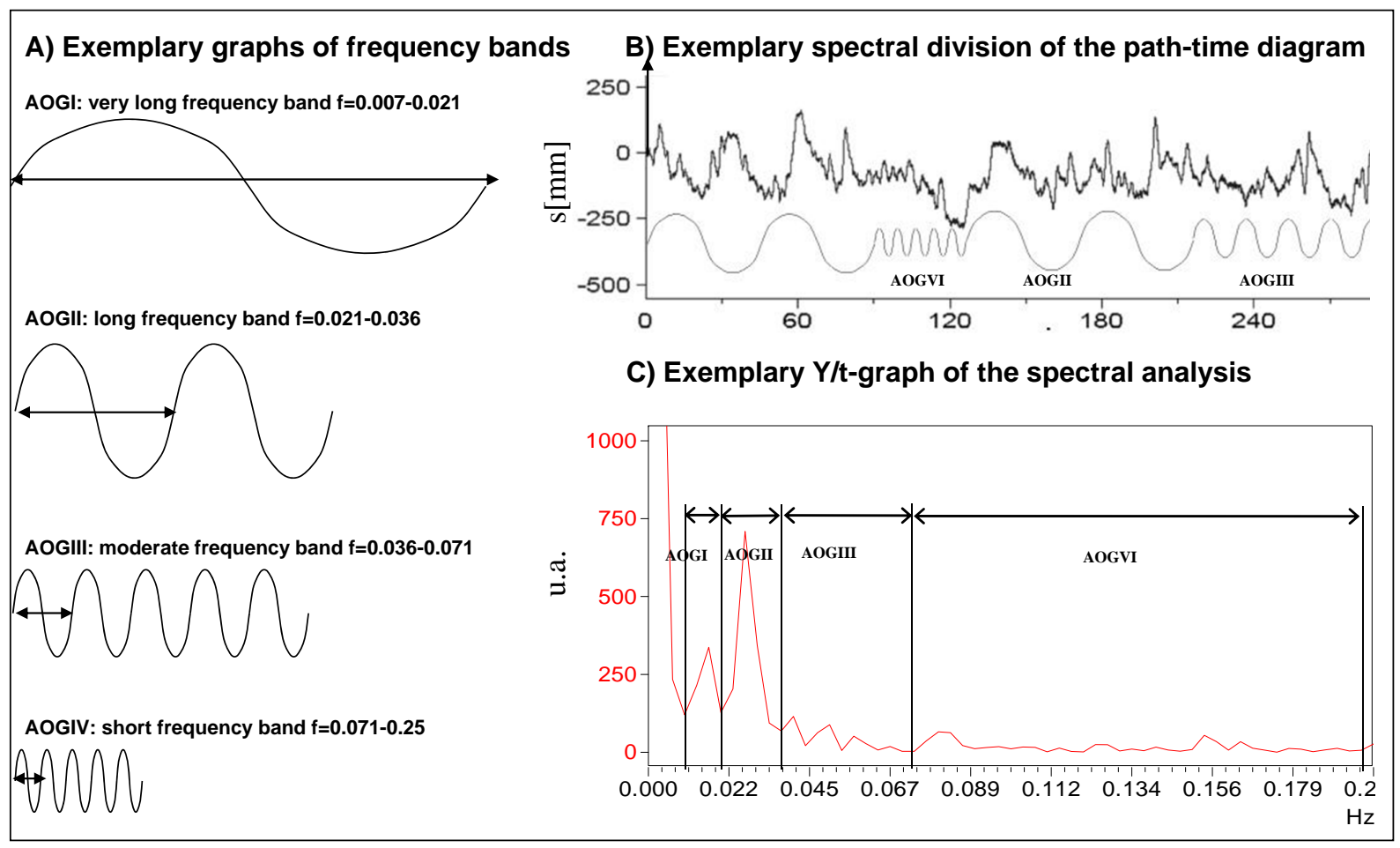

Fig. (3). A) Exemplary graphs of frequency bands AOG I: very long frequency band, AOG II: long frequency band, AOG III: moderate frequency band, AOG IV: short frequency band; B) Exemplary spectral division of path-time diagram; the spectral analysis divides a signal into sinus-frequency bands over the whole signal. this example shows a very simple idea, of how a complex signal can be divided into different sinus-signals. C) Exemplary Y/t graph of the spectral analysis We quantified the Power Spectral Density (PSD)-spectrum by integrating a) non-frequency weighted (AOG) and b) frequency-weighted after splitting the function into four frequency bands (AOG I-IV).

within AOG II. There were no significant differences for any frequency-weighted performance between the groups PDM and HC. Overall, the performance in gait adaptation in AOG II and III decreased from HC, to PDM, to PDS. AOG I and AOG IV did not differ between the groups.

\subsubsection{Gait Dynamics in PD Patients}

Table 3 shows the biomechanical gait parameters of all PD patients according to the spatiotemporal gait variables. These values were used for the correlation analyses.

\subsection{Correlation Results}

Table 4 summarizes the correlations between the parameters of the AOG-test and clinical parameters of the UPDRS test e.g. motor function, clinical gait analysis and biomechanical gait analysis.

AOG-all parameters showed correlations to UPDRS, UPDRS part III and a correlation to bradykinesia and cranial parameters. There was no correlation between AOG-all parameters and levodopa-dosage, freezing, falls, fluctuations, sensory dysfunction or dyskinesia. The webster-

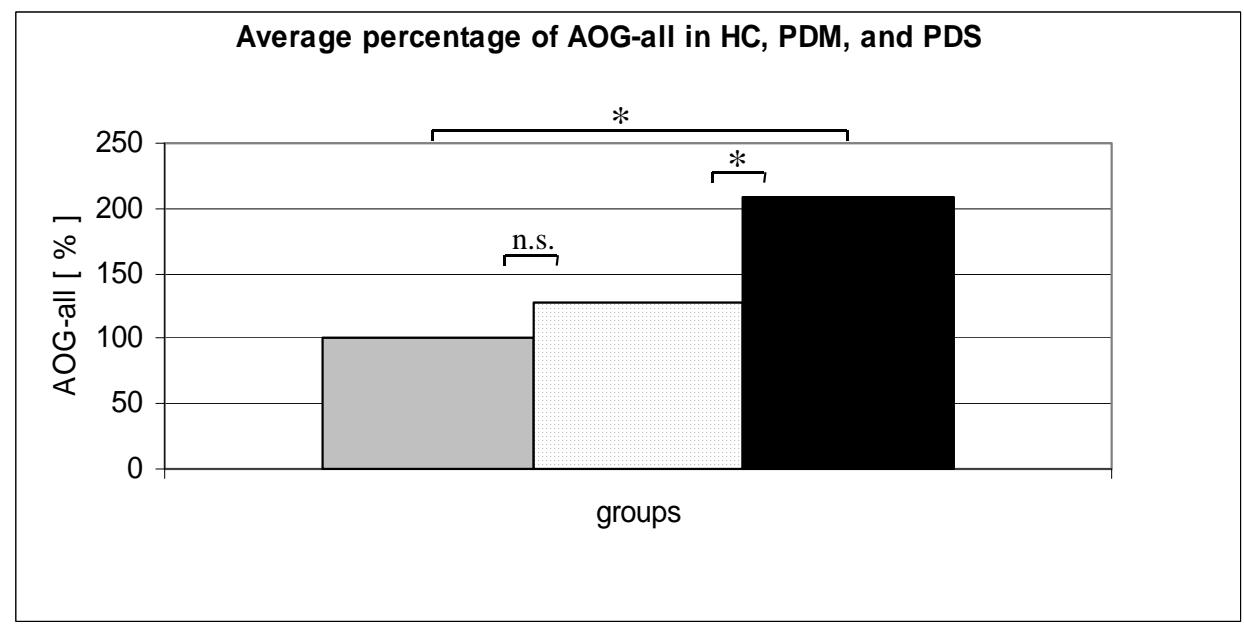

Fig. (4). Average percentage of non-frequency weighted performance in HC (grey bar), PDM (dotted bar) and PDS (black bar) (*: p<0.05; n.s: not significant). 
gait test had no correlations according to any AOGparameters. The spatiotemporal gait parameters e.g. double support time, stance phase or cycle time did not correlate with any AOG parameter.

Table 2. Outcome Measures

\begin{tabular}{|c|c|c|c|}
\hline & Mean (SD) & $\begin{array}{c}\text { ANOVA, } \\
\text { Between } \\
\text { Group, } \\
p \text { Value }\end{array}$ & $\begin{array}{c}\text { ANOVA, } \\
\text { Post-Hoc, } \\
\text { Pairwise } \\
\text { Comparisons, } \\
p \text { Value }\end{array}$ \\
\hline AOG-all & & \multirow{4}{*}{$0.015^{*}$} & \\
\hline PDM & $1.73(1.11)$ & & PDM vs PDS $0.021 *$ \\
\hline PDS & $2.82(1.64)$ & & PDS $v s$ HC $0.012 *$ \\
\hline $\mathrm{HC}$ & $1.35(0.76)$ & & $\mathrm{PDM} v s \mathrm{HC} 0.51$ \\
\hline AOG I (148-60sec) & & \multirow{4}{*}{0.104} & \\
\hline PDM & $0.93(1.07)$ & & \\
\hline PDS & $1.36(1.10)$ & & \\
\hline $\mathrm{HC}$ & $0.48(0.27)$ & & \\
\hline AOG II (60-28sec) & & \multirow{4}{*}{$0.002 * *$} & \\
\hline PDM & $0.23(0.12)$ & & PDM $v s$ PDS $0.001 * *$ \\
\hline PDS & $0.61(0.42)$ & & PDS $v s$ HC $0.02^{*}$ \\
\hline $\mathrm{HC}$ & $0.30(0.16)$ & & $\mathrm{PDM} v s \mathrm{HC} 0.595$ \\
\hline AOG III (28-14sec) & & \multirow{4}{*}{$0.069 t$} & \\
\hline PDM & $0.25(0.13)$ & & PDM $v s$ PDS $0.026^{*}$ \\
\hline PDS & $0.44(0.33)$ & & PDS $v s$ HC 0.174 \\
\hline $\mathrm{HC}$ & $0.30(0.23)$ & & $\mathrm{PDM} v s \mathrm{HC} 0.653$ \\
\hline AOG IV (14-4sec) & & \multirow{4}{*}{0.129} & \\
\hline PDM & $0.33(0.13)$ & & \\
\hline PDS & $0.41(0.17)$ & & \\
\hline $\mathrm{HC}$ & $0.27(0.24)$ & & \\
\hline
\end{tabular}

Results of ANOVA (analysis of variance) and post-hoc analysis according to AOG-all (parameter for the gait performance Adaptability-of-gait at the whole testing time) and AOGI-IV (subsequent parameters for the gait performance Adaptability-of-gait) between the groups PDM (Parkinson moderate), PDS (Parkinson severe) and HC (healthy control).

$\mathrm{p}<=0.01 * *$

$\mathrm{p}>0.01-0.05^{*}$

$0.01<\mathrm{p}<0.05 \mathrm{t}$.

More detailed information can be seen in the relations between the AOG frequency-weighted-parameters (AOG IIV). The total UPDRS score has the strongest correlation with the AOG II-parameter $(\mathrm{r}=0.631 ; p<0.01)$ and significant but moderate correlations to AOG-III. Motor function correlated moderately with AOG II-IV $(r>0.4 ; p<0.01)$. The subscales of motor function showed highly-significant correlations between AOG-II and rigidity, bradykinesia and cranial parameters. No correlation between gait and posture variables and AOG-frequency-weighted-parameter, as well as the tremor variable were detected. A moderate correlation was seen between $\mathrm{H} \& \mathrm{Y}$-stage and AOG, AOG II-III $(r=0.39 ; p<0.05)$

The frequency-weighted AOG I correlated not at all. Stride time showed for the velocities $1.5-2.1 \mathrm{~km} / \mathrm{h} \quad(r=0.44$; $p<0.01)$ and significant moderate correlations for the velocities 2.4 and $2.7 \mathrm{~km} / \mathrm{h}(r>0.32 ; p<0.05)$. Between step length and AOG III-parameter was a moderate negative relationship for all velocities (from $r=-0.35$ to $-0.43 ; p<0.05$ ) was detected.

\section{4, DISCUSSION}

The aim of this study was to develop and evaluate a method of biomechanical gait analysis which investigates the adaptability in gait to fluctuating external conditions: the AOG-test. Within our novel gait-test, velocities change periodically, but the magnitude and time of acceleration are unpredictable for the subjects. The test represents spontaneous adaptations during repetitive movements and therefore the adaptation through an ongoing process (walking). The AOG-test excludes start-stop situations e.g. gait-initiation, walk-to-stand, as well as chronic adaptationprocesses in the context of learning.

The main outcome is that the AOG-test identifies differences between PD-patients and $\mathrm{HC}$ according to the performance of gait adaptation. The results of AOG-all (performance in gait adaptation) of the group 'PD severe' (PDS) were significantly worse compared to the group 'PD moderate' (PDM) and 'healthy controls' (HC) (Table 2). Furthermore, AOG-all of PDM was $28 \%$ worse than HC, but the results did not reach significance (Fig. 4). These results indicate, that the performance in gait adaptation gets worse during disease progression (Table 1). This goes in line with most locomotor parameters. Gait parameters are related to the stages of the disease described in the clinical classification [3-11, 22].

To get detailed information about the causing gait differences, we correlated the AOG-test with existing, valid gait assessments and the UPDRS test. We found predominant no correlation between the AOG-test and these biomechanical or clinical tests, except one significant strong

Table 3. Gait Dynamics of the Study Population (PD, $n=36)$

\begin{tabular}{|c|c|c|c|c|c|c|}
\hline $\begin{array}{c}\text { Velocity } \\
(\mathbf{k m} / \mathbf{h})\end{array}$ & $\begin{array}{c}\text { Step Length } \\
(\mathbf{m})\end{array}$ & $\begin{array}{c}\text { Stride Time } \\
(\mathbf{s})\end{array}$ & $\begin{array}{c}\text { Stride Time Variability } \\
(\%)\end{array}$ & $\begin{array}{c}\text { Double Stance } \\
(\mathbf{m s})\end{array}$ & $\begin{array}{c}\text { Swing Time Left } \\
(\mathbf{m s})\end{array}$ & $\begin{array}{c}\text { Swing Time Right } \\
(\mathbf{m s})\end{array}$ \\
\hline \hline 1.5 & $.60(.10)$ & $1.43(.25)$ & $5.63(2.22)$ & $233(69)$ & $484(105)$ & $475(102)$ \\
\hline 1.8 & $.69(.11)$ & $1.35(.22)$ & $5.53(2.73)$ & $203(49)$ & $513(242)$ & $519(241)$ \\
\hline 2.1 & $.76(.11)$ & $1.29(.19)$ & $5.63(2.88)$ & $185(47)$ & $453(88)$ & $457(106)$ \\
\hline 2.4 & $.83(.11)$ & $1.24(.17)$ & $5.33(3.04)$ & $183(53)$ & $434(84)$ & $438(73)$ \\
\hline 2.7 & $.91(.11)$ & $1.2(.15)$ & $4.84(2.64)$ & $173(50)$ & $436(63)$ & $418(81)$ \\
\hline 3.0 & $.96(.12)$ & $1.16(.16)$ & $5.41(2.72)$ & $166(36)$ & $438(103)$ & $420(114)$ \\
\hline
\end{tabular}


Table 4. Correlation Between AOG Non Frequency Weighted and Frequency-Weighted Performance and UPDRS Subscales and Other Parkinsonian Features, Gait Parameter, Webster Gait Test and Gait Dynamics

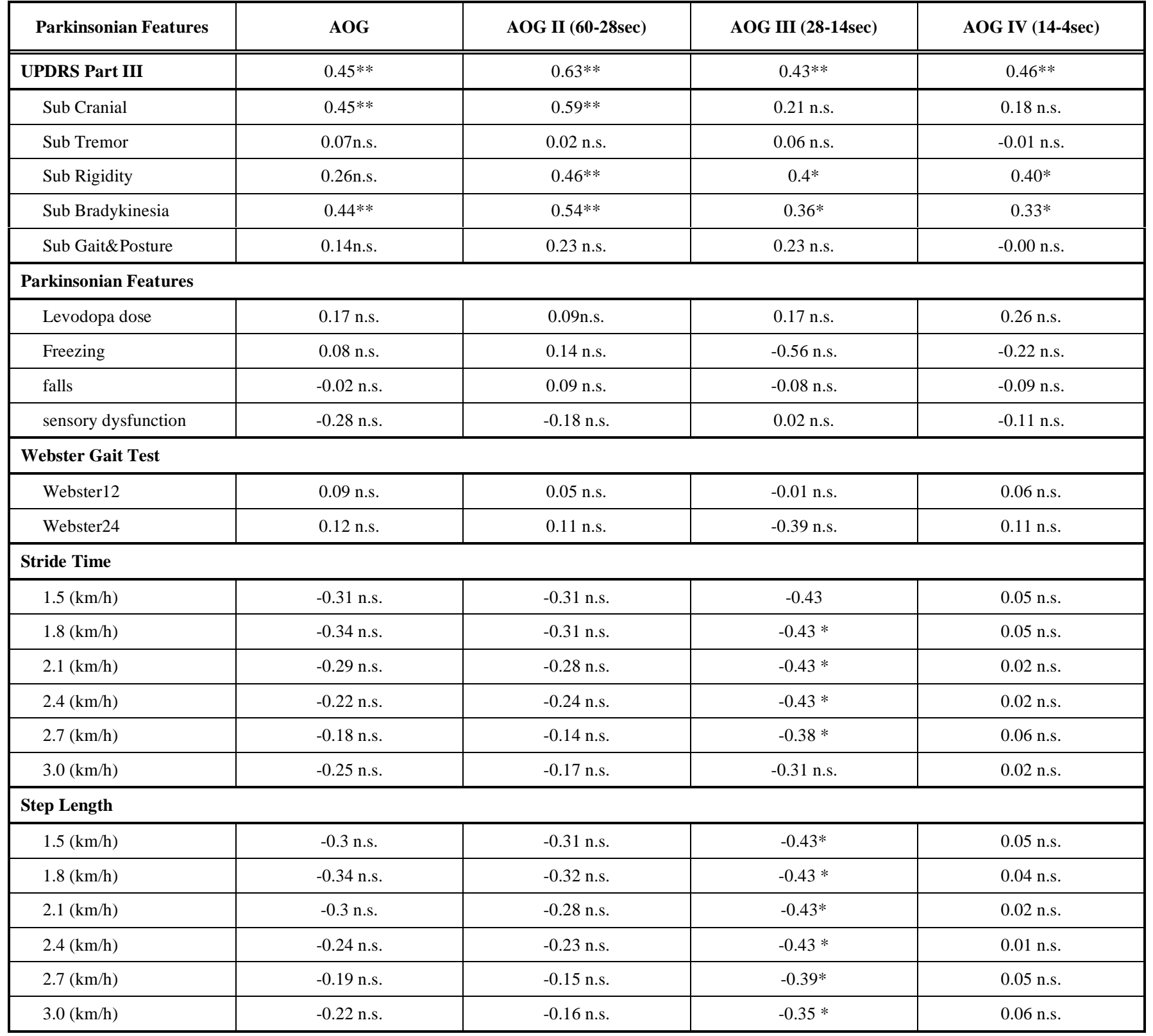

$\mathrm{p}<=0.01 * *$.

$0.01<\mathrm{p}<0.05^{*}$

p $>0.05$ n.s.

correlation with UPDRS bradykinesia and cranial subscale and moderate correlations with step frequency and steplength (see Table 4). Based on the fact that this study is of exploratory character, we decided to discuss strong and moderate correlations according to the AOG-test, to get first indications.

\subsection{Differing Gait Task}

In this study we used conventional clinical gait tests e.g. the UPDRS-test and the Webster gait-test, for comparison with the AOG-test. The UPDRS 'gait and posture' subscale includes the tasks 'standing up from a sitting position', 'the pull-test' (retropulsion-test) posture and 'gait in constant, self selected walking speed'. Gait is only one variable of four, which is examined over a short distance with constant velocity. By contrast, the AOG-test focusses on gait adaptation over a longer time and distance. Thus, the missing correlation to the 'gait and posture subscore' certified our presumption to have a completely different assessment-task in the AOG-test. This might explain the lack of correlation between the UPDRS 'gait and posture'-subscore and the adaptation of gait. Brusse et al. complain, that there is low evidence that the UPDRS-test adequately measures walking, mobility performance or comfortable and fast gait speeds [42].

These results are resembled by Turbanski et al. according to postural control. They also found no correlations between the UPDRS subscore 'gait and posture' and static or dynamic 
biomechanic tests for postural control. They concluded that the reliable retropulsion-test gives an insight into one phenomenon, and the dynamic test for postural control to another phenomenon. Nevertheless, both tests are important for the evaluation of postural control mechanisms in PD [43].

We took this into account, and used other qualitative (UPDRS), semi-quantitative (Webster-gait test) and quantitative gait analyses (AOG and biodynamic gait assessment). Others like the TUG (timed get up and go-test), BBS (Berg BalanceScale), FGA (Functional Gait Assessment) can also be used to measure gait performance [15, 42]. The Webster-test represents a gait task with initiation and constant gait elements at comfortable walking velocity and a turn. Although, the test inherits velocity changes, no correlation with the AOG-test can be seen. This might also be attributed 60 differing tasks. The Webster-gait test has feedforward processes while the AOG-test is characterized by an unpredictable feedback regulation. Also, gait has to be adapted more frequently than in the Webster-gait test and other common clinical gait assessments.

\subsection{Adaptation of Gait and Bradykinesia}

Insights into the dominating parameters of gait adaptation get visible by examining the correlation with the cardinal symptom bradykinesia. Bradykinesia includes the terms hypokinesia and akinesia. Several studies analyzed and described the influence of hypokinesia to gait $[3,7,17,18]$. Hypokinesia refers to slow movements e.g. slowed gait velocity and smaller amplitudes e.g. shortened step length [41]. Akinesia is defined as a poverty of spontaneous movements, like sudden changes in gait velocity or facial expression [41].

Both hypokinesia and akinesia can be part of the gait adaptation process. Continuous slow and short stepped gait reasoned by hypokinesia would have caused a back drift on the treadmill during the AOG-test, in particular during the fast velocities and the positive velocity changes $(+0.5 \mathrm{~km} / \mathrm{h})$ in the test. This would have caused a higher, probably significant value in AOG-IV, representing difficulties in adapting to one, respectively two velocity steps. Controversially, a back drift might also have been compensated and balanced through the tests velocity-time sequence (see Fig. 2). We chose a range of treadmill-velocities between 1.2 and $3.8 \mathrm{~km} / \mathrm{h}$ which lies beneath the average gait-velocity of PD patients at this age and disease stadium. So we assume, that hypokinesia should play a subordinate role in the AOG-test, explaining the lack of the correlation.

Another aspect was described by Morris et al. [44]. They argued, that gait hypokinesia is responsible for an impaired stride length, so that PD subjects compensate their impaired stride length with a higher stride time, a parameter that is not impaired in PD-gait $[6,9,17,24]$. Stride time might have compensated the reduced step length during the whole AOGtest. According to some velocity steps in the AOG-test, this regulation-pattern seems to get critical in several adaptation situations, referred to the subjects constant position on the treadmill, like the correlation between AOG III and stride length and stride time shows. Stride length and stride time seem not to be able to compensate the fluctuating gait-velocities adequately. Nevertheless, the results have moderate correlations in all velocities.

Akinesia might explain differences in gait adaptation. Changes of velocity (+/-) during the AOG-test are getting critical, because akinetic PD patients have a poverty of spontaneous movements in both directions: undershooting movements during a positive velocity change, overshooting movements during a negative velocity change. Further studies found hyperstability of walking coordination that leads to difficulties in adapting to a single velocity change during gait on a treadmill [45]. Winogrodzka et al. showed that rigidity and bradykinesia limited gait-parameters significantly in PD patients, depending on the degeneration of the dopamine system. They analyzed the ability to adapt to single, signalized velocity-change on the treadmill. Bradykinesia was significantly associated with the coordination of arm and leg movements during this walking-task (velocity change plus and minus $0.8 \mathrm{~km} / \mathrm{h}$ between 0.6 and $5.4 \mathrm{~km} / \mathrm{h}$, one signalized step every 60 -second), so that the gait adaptation was retarded. They conclude that the extent of degeneration of the dopamine system was associated with a limited adaptive ability in movement coordination [23]. This goes in-line with our study, where bradykinesia correlated with the AOG parameters (moderately), and the progression in PD (highly).

Interesting in this context is the significantly high correlation $(\mathrm{r}=0.59 ; \mathrm{p}<0.000)$ between AOG II and the cranial subscale. In our study the items 'speech' and 'facial expression' show the highest association of all analyzed parameters to the AOG-test. The high correlation between the cranial-subscale and the AOG-test might be caused by akinesia, because both are caused by poverty of spontaneous movements $[41,46]$. Cantiniaux et al. were the first who studied correlations between speech and gait parameters. Their quantitative examination of gait and speech parameters correlated significantly in $\mathrm{HC}$ and $\mathrm{PD}$ subjects [47]. They found parallels in the regulation of gait and speech in HC and PD subjects. Walking velocity and speech velocity, as well as step length and the so-called ISD (time interval between two pauses) significantly correlated, levodopa dependent. Furthermore, the rhythmic-parameter of gait and speech: walking cadence and the so-called SPIR (Speech Index of Rhythmic) correlated (negative) significantly in $\mathrm{HC}$ and in PD off-stage and could not be influenced with levodopa. Speech or facial expression and gait are both complex mechanisms in different non-dopaminergic structures [47]. Cantiniaux et al. bring out the possibility of a speech rhythm generator comparable to the gait rhythm generators that are well-known through numerous studies regarding spinal cord injuries (human and animal based). Repeated rhythmic stimulation of the lower extremities leads to the activation of the so called centralpattern-generators (CPG) [48], which are networks of spinal nerve cells that work self-sufficient after activation and lead to a rhythmic and functional activation of agonistic and antagonistic leg muscles during gait [49-51]. Nevertheless they are bound to proprioceptic and exteroceptic afferences. So even if priorities change within the integration of sensoric modalities, the processing of proprioceptic information should still play a major role during the generation of a rhythmic gait pattern. In humans the discreteness of the CPG is influenced by supraspinal areas [52]. A major structure of the supraspinal regulation is the mesencephylic locomotor region (MLR) which is connected with the pedunculopontine nucleus (PPN) via colineric neurons [53]. The PPN is sending sensoric feedback to the basal ganglia [54] as well as efferences to the spinal cord. The PPN shows a rhythmic activity which is controlled through cerebellar paths. In PD-patients a reduced amount of cells in the PPN was found in postmortem [48, 55]. Because the neurons of the PPN are 
dopamin-independent, a degeneration of the PPN neurons could be one of the causes for dopamin-resistent PD-symptoms, like freezing of gait, postural instability or sleep disorders [56].

Relating to our study where all PD-patients were tested pharmaceutically "on", the significant worse gait adaption of the PDS-group in the AOG-test could possibly be caused by a malfunction of this dopamin-independent interaction. In consequence of a nerve cell loss in the PPN, the supraspinal efferences from the PPN to the CPG could potentially be defective when state changes of velocity take place. It seems that an ongoing adaptation of the gait velocity is not possible, because the feedback with the basal ganglia is limited as well in the worse affected group PDS. A further hint for this explanation gives the results of Getrost [57] in an additional study with the AOG-test. He found pecularities regarding PDpatients with freezing of gait (FOG) relating to the adaptability of gait in comparison to patients without FOG and patients with dyskinesia.

In summary: the AOG-test clearly makes restrictions in gait adaption apparent. Yet the underlying pathologic mechanisms or interfaces became not fully evident. Our analyzation of data and literature brings us to considerations that a defective gait adaptability is potentially associated with akinesia and/or dopamin-independent alterations in the sensorimotor integration between PPN and CPG.

\section{CONCLUSION}

It is obvious, that the gait task intentionally differs from the conventional gait-tests. Gait adaptation seems to refer partly to the cardinal symptom bradykinesia, but the main influencing part is still unclear. Thus, the AOG-test obviously exposes different phenomena we cannot allocate yet. Several explanations are possible, e.g. impaired sensorimotor integration, hypokinesia, akinesia or levodopa induced dyskinesia. Further evaluation processes are necessary to improve the diagnostics like different patient groups (freezer/non-freezer; "on"/"off" state) or additive assessments (speech and cognitive variables, e.g. attention). There also seems to be a potential of the AOG-test as a therapy strategy. Different studies have already shown that deviance based gait training and treadmill training are effective interventions to improve pathological gait [58, 59]. The AOG-test combines those two approaches and could be useful as a training tool.

To our knowledge, this is the first test that explores the adaptability of gait. The detected parameters are not represented in the usual biomechanical and clinical gait assessments to date. Whereas it is a well known difficulty of PD subjects to adapt to changing gait conditions (e.g. at crowded places). We conclude, that the AOG-test seems to measure a new and important factor in gait-regulation and might represent a dynamic gait-task that detects gait deficits influenced by akinesia and/or a dopaminindependent phenomenon relying on a defective processing in the gait rhythm.

\section{CONFLICT OF INTEREST}

The authors confirm that this article content has no conflict of interest.

\section{ACKNOWLEDGEMENTS}

We gratefully thank Priv.-Doz. Dr. PhD. Iris Reuter for medical leadership, and the essential ethical decisions. We also thank: Prof. Dr. Stephan Turbanski and Sports Scientist Carsten Ebert for helpful discussions and review of the manuscript. We gratefully thank all patients and healthy subjects who participated in this study.

This research was partly funded by the Soemmerring Institute Bad Nauheim, Germany.

The study was supported by the ethic committee of the Justus Liebig University in Giessen, Germany, human science in consideration of the ICH-GCP, no. 88/05 accepted: 11.8.2005.

\section{REFERENCES}

[1] Hirtz D, Thurman DJ, Gwinn-Hardy K, Mohamed M, Chaudhuri AR, Zalutsky R. How common are the "common'" neurologic disorders? Neurology 2007; (68): 326-37.

[2] Hausdorff JM. Gait dynamics in Parkinson's disease: Common and distinct behavior among stride length, gait variability, and fractallike scaling. Chaos 2009; 19(2): 26113.

[3] Morris ME, Huxham F, McGinley J, Dodd K, Iansek R. The biomechanics and motor control of gait in Parkinson disease. Clin Biomech 2001; 16(6): 459-70.

[4] Murray MP. Gait as a total pattern of movement. Am J Phys Med 1967; 46(1): 290.

[5] Knutsson E. An analysis of parkinsonian gait. Brain 1972; 95(3): 475.

[6] Blin O, Ferrandez AM, Serratrice G. Quantitative analysis of gait in Parkinson patients: increased variability of stride length. J Neurol Sci 1990; 98(1): 91-7.

[7] Morris ME, Iansek R, Matyas TA, Summers JJ. The pathogenesis of gait hypokinesia in Parkinson's disease. Brain 1994; 117(5): 1169.

[8] Hausdorff JM, Schaafsma JD, Balash Y, Bartels AL, Gurevich T, Giladi N. Impaired regulation of stride variability in Parkinson's disease subjects with freezing of gait. Exp Brain Res 2003; 149(2): 187.

[9] Schaafsma JD, Giladi N, Balash Y, Bartels AL, Gurevich T, Hausdorff JM. Gait dynamics in Parkinson's disease: relationship to Parkinsonian features, falls and response to levodopa. J Neurol Sci 2003; 212(1-2): 47-53.

[10] Frenkel-Toledo S, Giladi N, Peretz C, Herman T, Gruendlinger L, Hausdorff JM. Effect of gait speed on gait rhythmicity in Parkinson's disease: variability of stride time and swing time respond differently. J Neuroeng Rehabil 2005; 2(1): 23.

[11] Hausdorff JM. Gait dynamics, fractals and falls: finding meaning in the stride-to-stride fluctuations of human walking. Hum Mov Sci 2007; 26(4): 555-89.

[12] Bessou P, Dupui P, Montoya R, Pages B. Simultaneous recording of longitudinal displacements of both feet during human walking. J Physiol 1988-1989; 83(2): 102-10.

[13] Ebersbach G, Sojer M, Valldeoriola F, et al. Comparative analysis of gait in Parkinson's disease, cerebellar ataxia and subcortical arteriosclerotic encephalopathy. Brain 1999; 122(7):1349.

[14] Morris ME, McGinley J, Huxham F, Collier J, Iansek R Constraints on the kinetic, kinematic and spatiotemporal parameters of gait in Parkinson's disease. Hum Mov Sci 1999; 18(2-3): 461-83.

[15] Schwed M, Kersten S, Scholl N, Haas C. Assessment von neurologischen Gangstörungen Assessment of neurological gait disorders. B \& G 2009; 25(1): 14-23.

[16] Reuter I, Mehnert S, Leone P, Kaps M, Oechsner M, Engelhardt M. Effects of a Flexibility and Relaxation Programme, Walking, and Nordic Walking on Parkinson's Disease. J Aging Res 2011; 2011: 232473.

[17] Morris ME, Iansek R, Matyas TA, Summers JJ. Ability to modulate walking cadence remains intact in Parkinson's disease. J Neurol Neurosurg Psychiatr 1994; 57(12): 1532.

[18] Morris ME, Iansek R, Matyas TA, Summers JJ. Stride length regulation in Parkinson's disease: normalization strategies and underlying mechanisms. Brain 1996; 119(2): 551.

[19] Frenkel-Toledo S, Giladi N, Peretz C, Herman T, Gruendlinger L, Hausdorff JM. Treadmill walking as an external pacemaker to improve gait rhythm and stability in Parkinson's disease. Mov Disord 2005; 20(9): 1109-14. 
[20] Hausdorff JM, Cudkowicz ME, Firtion R, Wei JY, Goldberger AL. Gait variability and basal ganglia disorders: Stride to stride variations of gait cycle timing in parkinson's disease and Huntington's disease. Mov Disord 1998; 13(3): 428-37.

[21] Hausdorff JM, Rios DA, Edelberg HK. Gait variability and fall risk in community-living older adults: A 1-year prospective study. Arch Phys Med Rehabil 2001; 82(8): 1050-6.

[22] Carpinella I, Crenna P, Calabrese E, et al. Locomotor function in the early stage of Parkinson's disease. Neural Syst Rehabil Eng 2007; 15(4): 543-51

[23] Winogrodzka A, Wagenaar RC, Booij J, Wolters EC. Rigidity and bradykinesia reduce interlimb coordination in Parkinsonian gait. Arch Phys Med Rehabil 2005; 86(2): 183-9.

[24] Hely MA, Morris JG, Traficante R, Reid WG, O'Sullivan DJ, Williamson PM. The Sydney multicentre study of Parkinson's disease: progression and mortality at 10 years. J Neurol Neurosurg Psychiatr 1999; 67(3): 300.

[25] Hausdorff JM. Gait variability: methods, modeling and meaning. J Neuroeng Rehabil 2005; 2(1): 19.

[26] Azulay JP, van Den Brand C, Mestre D, et al. Automatic motion analysis of gait in patients with Parkinson disease: effects of levodopa and visual stimulations. Rev Neurol 1996; 152(2): 128

[27] Rochester L, Hetherington V, Jones D, et al. The effect of external rhythmic cues (auditory and visual) on walking during a functional task in homes of people with Parkinson's disease. Arch Phys Med Rehabil 2005; 86(5): 999-1006.

[28] Bloem BR, Am Grimbergen Y, Cramer M, Willemsen M, Zwinderman AH. Prospective assessment of falls in Parkinson's disease. J Neurol 2001; 248(11): 950-8.

[29] Lim L, van Wegen EE, Goede CJ de, et al. Measuring gait and gait-related activities in Parkinson's patients own home environment: a reliability, responsiveness and feasibility study. Parkinsonism Relat Disord 2005; 11(1): 19-24.

[30] Carpinella I, Crenna P, Rabuffetti M, Ferrarin M. Coordination between upper-and lower-limb movements is different during overground and treadmill walking. Eur J Appl Physiol 2010; 108(1): 71-82

[31] Leuchte S, Stöber K. Laufband versus Gangbahn? - Theorie, Methodik und Empirie. LSB (Sankt Augustin) 2002; 43(1): 62-86.

[32] Lee SJ, Hidler J. Biomechanics of overground vs. treadmill walking in healthy individuals. J Appl Physiol 2008; 104(3): 74755 .

[33] Murray MP, Spurr GB, Sepic SB, Gardner GM, La Mollinger. Treadmill vs floor walking: kinematics, electromyogram, and heart rate. J Appl Physiol 1985; 59(1): 87-91.

[34] Nymark JR, Balmer SJ, Melis EH, Lemaire ED, Millar S. Electromyographic and kinematic nondisabled gait differences at extremely slow overground and treadmill walking speeds. J Rehabil Res Dev 2005; 42(4): 523.

[35] White SC, Yack HJ, Tucker CA, Lin HY. Comparison of vertical ground reaction forces during overground and treadmill walking. Med Sci Sports Exerc 1998; 30(10): 1537.

[36] Goldberg EJ, Kautz SA, Neptune RR. Can treadmill walking be used to assess propulsion generation? J Biomech 2008; 41(8): $1805-8$.

[37] Regnaux JP, Robertson J, Smail DB, et al. Human treadmill walking needs attention. J Neuroeng Rehabil 2006; 3: 19.

[38] Riley PO, Paolini G, Della Croce U, Paylo KW, Kerrigan DC. A kinematic and kinetic comparison of overground and treadmill walking in healthy subjects. Gait Posture 2007; 26(1): 17-24.
[39] Fahn S, Elton RL. Members of the UPDRS Development Committee. Unified Parkinson's disease rating scale. Rec Dev Parkinson' Dis 1987; 2(3): 153-64.

[40] Webster DD. Critical analysis of the disability in Parkinson's disease. Mod Treat 1968; 5(2): 257-82.

[41] Berardelli A, Rothwell JC, Thompson PD, Hallett M. Pathophysiology of bradykinesia in Parkinson's disease. Brain 2001; 124(11): 2131

[42] Brusse KJ, Zimdars S, Zalewski KR, Steffen TM. Testing functional performance in people with Parkinson disease. Phys Ther 2005; 85(2): 134

[43] Turbanski S, Haas CT, Schmidtbleicher D, Friedrich A, Duisberg P. Effects of random whole-body vibration on postural control in Parkinson's disease. Res Sports Med 2005; 13(3): 243-56.

[44] Morris ME, Iansek R. Characteristics of motor disturbance in Parkinson's disease and strategies for movement rehabilitation. Hum Mov Sci 1996; 15(5): 649-69.

[45] van Emmerik REA, Wagenaar RC, Winogrodzka A, Wolters EC Identification of axial rigidity during locomotion in parkinson disease. Arch Phys Med Rehabil 1999; 80(2): 186-91.

[46] Schulz GM, Grant MK. Effects of speech therapy and pharmacologic and surgical treatments on voice and speech in parkinson's disease:: A review of the literature. J Commun Disord 2000; 33(1): 59-88.

[47] Cantiniaux S, Vaugoyeau M, Robert D, T et al. Comparative analysis of gait and speech in Parkinson's disease: hypokinetic or dysrhythmic disorders? J Neurol Neurosurg Psychiatr 2010; 81(2): 177.

[48] Pahapill PA, Lozano AM. The pedunculopontine nucleus and Parkinson's disease. Brain 2000; 123(9): 1767-83.

[49] Dietz V. Spinal cord pattern generators for locomotion. Clin Neurophysiol 2003; 114(8): 1379-89.

[50] Pearson KG. Neural adaptation in the generation of rhythmic behavior. Ann Rev Physiol 2000; 62(1): 723-3.

[51] Wernig A, Müller S, Nanassy A, Cagol E. Laufband therapy based on rules of spinal locomotion is effective in spinal cord injured persons. Eur J Neurosci 1995; 7(4): 823-9.

[52] Ebersbach G, Sojer M, Valldeoriola F, et al. Comparative analysis of gait in Parkinson's disease, cerebellar ataxia and subcortical arteriosclerotic encephalopathy. Brain 1999; 122(7): 1349.

[53] Garcia-Rill E. The basal ganglia and the locomotor regions. Brain Res Rev 1986; 11(1): 47-63

[54] Mena-Segovia J, Bolam JP, Magill PJ. Pedunculopontine nucleus and basal ganglia: distant relatives or part of the same family? Trends Neurosci 2004; 27(10): 585-8.

[55] Zweig RM, Jankel WR, Hedreen JC, Mayeux R, Price DL. The pedunculopontine nucleus in Parkinson's disease. Ann Neurol 1989; 26(1): 41-6.

[56] Bartels AL, Leenders KL. Brain imaging in patients with freezing of gait. Mov Disord 2008; 23(S2): S461-7.

[57] Getrost T. Trial of an instrument-based gaittest for the analysis of neurologic disease patterns (at the example Parkinson's disease). Frankfurt a. M.: Univ.-Bibliothek Frankfurt am Main 2006.

[58] Haas CT, Kersten S, Lutz C, Schwed M. Deviance-based gait training in Multiple Sclerosis: 6th Congress of the European Interdisciplinary Society for Clinical and Sports Application. Isocinetics Exerc 2010; 18(1): 82-3.

[59] Herman T, Giladi N, Gruendlinger L, Hausdorff JM. Six weeks of intensive treadmill training improves gait and quality of life in patients with Parkinson's disease: a pilot study. Arch Phys Med Rehabil 2007; 88(9): 1154-8. 